\title{
Cow Urine: Plant Growth Enhancer and Antimicrobial Agent
}

\author{
Karthikeya Srinivasa Varma Gottimukkala ${ }^{1, a}$, Bishwambhar Mishra ${ }^{2, b^{*}}$, \\ Sampadha Joshi ${ }^{1, \mathrm{c}}$, Madhu Karan Reddy ${ }^{1, \mathrm{~d}}$ \\ 1Department of Biotechnology, Sreenidhi Institute of Science and Technology, Yamnampet, \\ Ghatkesar, India 501301 \\ ${ }^{2}$ Chaitanya Bharathi Institute of Technology, Gandipet, Hyderabad, Telangana, India, 500075 \\ akarthikeya.varma@icloud.com, bmishra.bishwambhar@gmail.com, csampadha05@gmail.com, \\ dmadhunani28ma@gmail.com
}

\section{Keywords: Antimicrobial, Cow urine, GCMS analysis, Plant Growth Enhancer.}

\begin{abstract}
The cow is considered a divine animal in the Hindu culture. The milk, cow dung, urine of the cow is used for various purposes. The benefits of cow urine have been depicted in ancient Hindu texts, Vedas. The present study aimed at using cow urine as a plant growth enhancer and antimicrobial agent. The plants chosen for this study were Zea mays (maize), Triticum aestivum (wheat), Cymbopogon citratum (grass), Ocimum basilicum (tukmaria) and Trigonella foenum graecum (methi). The plants were grown for 30 days using different cow urine concentrations i.e $0 \%$ (control), $5 \%, 15 \%, 25 \%$. The various parameters such as plant height, shoot, and root length, number of leaves, the mass of the root etc were observed. The antibacterial test using different cow urine concentrations i.e $0 \%$ (control), $5 \%, 15 \%, 25 \%$ was conducted on Escherichia coli using disc diffusion method. The fungus was screened and isolated from raw coconut and was grown on YPD media to obtain the mother culture. Using Slide culture technique, the fungi were identified as Aspergillus. The media was poisoned using different cow urine concentrations i.e $0 \%$ (control), $5 \%, 15 \%, 25 \%$ and the fungi culture was inoculated. GCMS analysis was conducted to identify the compounds present in the cow urine. Among the concentrations, 5\% cow urine concentration showed maximum growth when compared to other concentrations whereas $25 \%$ concentration showed more antibacterial and antifungal activity when compared to others. In GCMS Analysis, 16 compounds have been identified, in which, 6 compounds were antifungal, 3 compounds were antibacterial, and 2 compounds as plant growth enhancers. This study concludes that cow urine can be used as a plant growth enhancer and it possesses antimicrobial characteristics.
\end{abstract}

\section{Introduction}

The urine of Cow is commonly used to prevent and cure diseases especially by the rural people in India. Cow is considered sacred among the Hindu religion in India since ages. The use of Cow urine diminished when the western medicine has been developed, although it is still practiced as a treatment in some parts of the world. Currently, the use of Cow Urine as a therapeutic agent is being practiced to treat diseases and prevent the spread of diseases. Nonetheless, not much of the composition of the Cow Urine and its functions are known.

In Vedas (Ancient Hindu texts), Cow Urine has been given a lot of significance. It was also called as Amrita, the beverage of immortality. The Cow Urine from the Indian cow breed has medicinal properties unlike the crossbred or exotic cows. Recent studies have showed that Cow Urine has an effect on the immune system by increasing the phagocytic activity of macrophages. It was also shown that it has anti-bacterial properties [1]. Some medicinal properties of Cow Urine include reduction of weight, treating kidney problems, treating indigestion and edema [2]. The practitioners of Buddhist culture in Myanmar still use Cow Urine as a traditional medicine [3].

The constituents of the Cow Urine in right proportions are the reason to cure diseases. The analysis of Cow Urine showed that it is made up of all the essential Salts, Metals, Minerals, Vitamins, Enzymes and other entities in small quantities [4]. 
In this study, we tried to identify if there is any effect on the Plant growth in the presence of Cow Urine. The Antimicrobial activity of the Cow Urine was also studied. The Cow Urine was also analyzed using Gas Chromatography-Mass Spectroscopy (GCMS) to determine its constituents and its effects.

\section{Materials and Methods}

\section{Cow urine collection}

The cow urine was collected in a nearby organic farm and was filtered using filter paper. The urine was collected in urine sample kits and was stored at 4 Celsius. The soil was also collected from the organic farm.

\section{Seed collection}

5 types of seeds were collected i.e
1. Zea mays - Maize
2. Triticum aestivum - Wheat
3. Trigonella foenum graecum - Methi
4. Ocimum basilicum - Tukmaria (Sabja)
5. Cymbopogon citratus - Lemon grass

\section{Preparation of different cow urine concentrations}

Various concentrations of cow urine were prepared i.e $0 \%, 5 \%, 15 \%, 25 \%$.

$5 \%$ cow urine was prepared by mixing $5 \mathrm{ml}$ in $100 \mathrm{ml}$ distilled water.

$15 \%$ cow urine was prepared by mixing $15 \mathrm{ml}$ in $100 \mathrm{ml}$ distilled water.

$25 \%$ cow urine was prepared by mixing $25 \mathrm{ml}$ in $100 \mathrm{ml}$ distilled water.

\section{Sowing of seeds}

The seeds were sown in pots and were labeled using small flags which depict the name of the seed and the concentration of the cow urine used as shown in the Fig. 1. The plants were irrigated using different concentrations of cow urine $(10 \mathrm{ml})$ for 30 days and the growth was observed as shown in Fig. 1.

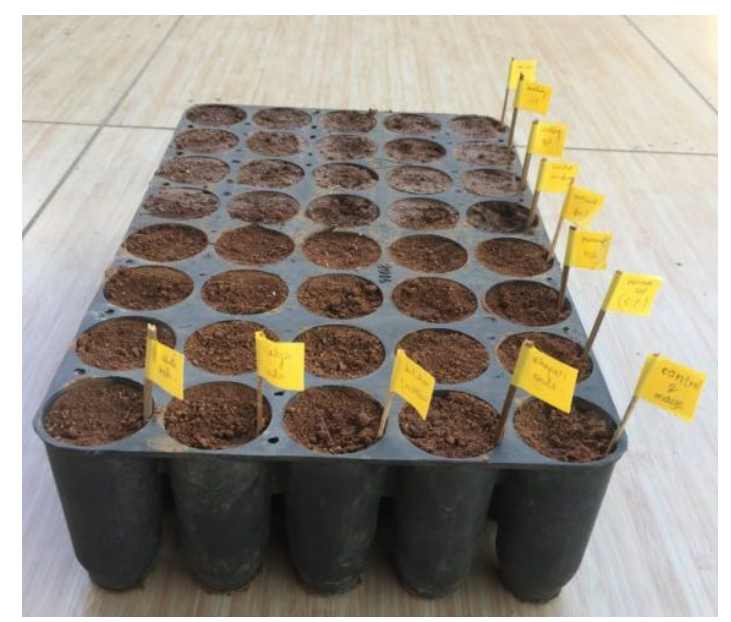

Figure 1. Seeds sown in their respective blocks

\section{Parameters}

Various parameters of the plants were observed. The parameters include

1. Total chlorophyll [5]:

Chlorophyll was extracted using acetone and spectrophotometer was used to measure the absorbance at specific wavelengths i.e $663 \mathrm{~nm}$ and $645 \mathrm{~nm}$.

Chlorophyll a $(\mu \mathrm{g} / \mathrm{ml})=12.7\left(\mathrm{~A}_{663}\right)-2.69\left(\mathrm{~A}_{645}\right)$

Chlorophyll b $(\mu \mathrm{g} / \mathrm{ml})=22.9\left(\mathrm{~A}_{645}\right)-4.68\left(\mathrm{~A}_{663}\right)$

Total Chlorophyll $(\mu \mathrm{g} / \mathrm{ml})=20.2\left(\mathrm{~A}_{645}\right)-8.02\left(\mathrm{~A}_{663}\right)$

2. Plant height: Measured using scale in $\mathrm{cms}$. 
3. Root Length: Measured from the shoot ending to the root tip using scale.

4. Shoot Length: Measured shoot using scale in cms.

5. Leaf Length: The leaf with highest length was considered.

6. Leaf breadth: The breadth of the highest leaf length was considered.

7. No of branches

8. Root weight: dried weight(gm) of the root is considered.

\section{Soil analysis}

The soil samples is given for analysis in ECOICONS, ECIL, to determine what changes have been made by the cow urine in the soil. Various parameters and methods used for soil analysis are tabulated in Table 1.

Table 1. The parameters measured in the soil analysis

\begin{tabular}{|c|c|c|c|}
\hline S.No & Parameters & Method & Units \\
\hline 1 & $\mathrm{pH}$ & IS:2720 (Part-26):2011 & - \\
\hline 2 & Electrical Conductivity & $\begin{array}{l}\text { Method Manual, Soil Testing, Ministry } \\
\text { of Agriculture, Govt of India }\end{array}$ & micro mhos \\
\hline 3 & Bulk Density & IS:2720 (Part-28):2011 & $\mathrm{g} / \mathrm{cc}$ \\
\hline 4 & Moisture content & IS:2720 (Part-18):2002 & $\%$ \\
\hline 5 & Soil Texture-Sand & Sieve Method & $\%$ \\
\hline 6 & Soil Texture-Silt & Sieve Method & $\%$ \\
\hline 7 & Soil Texture-Clay & Sieve Method & $\%$ \\
\hline 8 & Nitrogen & - & $\mathrm{kg} / \mathrm{ha}$ \\
\hline 9 & Phosphorus as $\mathrm{P} 2 \mathrm{O} 5$ & $\begin{array}{l}\text { Method Manual, Soil Testing, Ministry } \\
\text { of Agriculture, Govt of India }\end{array}$ & $\mathrm{kg} / \mathrm{ha}$ \\
\hline 10 & Potassium as $\mathrm{K} 2 \mathrm{O}$ & $\begin{array}{l}\text { Method Manual, Soil Testing, Ministry } \\
\text { of Agriculture, Govt of India }\end{array}$ & $\mathrm{kg} / \mathrm{ha}$ \\
\hline 11 & Sodium as $\mathrm{Na} 2 \mathrm{O}$ & $\begin{array}{c}\text { Method Manual, Soil Testing, Ministry } \\
\text { of Agriculture, Govt of India }\end{array}$ & $\mathrm{mg} / \mathrm{kg}$ \\
\hline 12 & Calcium as $\mathrm{Ca}$ & $\begin{array}{c}\text { Method Manual, Soil Testing, Ministry } \\
\text { of Agriculture, Govt of India }\end{array}$ & $\mathrm{mg} / \mathrm{kg}$ \\
\hline 13 & Magnesium as Mg & $\begin{array}{c}\text { Method Manual, Soil Testing, Ministry } \\
\text { of Agriculture, Govt of India }\end{array}$ & $\mathrm{mg} / \mathrm{kg}$ \\
\hline 14 & Chlorides as $\mathrm{Cl}$ & $\begin{array}{l}\text { Method Manual, Soil Testing, Ministry } \\
\text { of Agriculture, Govt of India }\end{array}$ & $\mathrm{mg} / \mathrm{kg}$ \\
\hline 15 & Sulphates as SO4 & $\begin{array}{l}\text { Method Manual, Soil Testing, Ministry } \\
\text { of Agriculture, Govt of India }\end{array}$ & $\mathrm{mg} / \mathrm{kg}$ \\
\hline 16 & Copper as $\mathrm{Cu}$ & $\begin{array}{c}\text { USEPA:3050B, \& Copper by Flame } \\
\text { AAS } \\
\end{array}$ & $\mathrm{mg} / \mathrm{kg}$ \\
\hline 17 & Manganese & $\begin{array}{c}\text { USEPA:3050B, \& Manganese by Flame } \\
\text { AAS }\end{array}$ & $\mathrm{mg} / \mathrm{kg}$ \\
\hline 18 & Molybdenum & $\begin{array}{l}\text { Method Manual, Soil Testing, Ministry } \\
\text { of Agriculture, Govt of India }\end{array}$ & $\mathrm{mg} / \mathrm{kg}$ \\
\hline 19 & Boran & $\begin{array}{l}\text { Method Manual, Soil Testing, Ministry } \\
\text { of Agriculture, Govt of India }\end{array}$ & $\mathrm{mg} / \mathrm{kg}$ \\
\hline 20 & Zinc as $\mathrm{Zn}$ & USEPA:3050B, \& Zinc by Flame AAS & $\mathrm{mg} / \mathrm{kg}$ \\
\hline 21 & Chromium as $\mathrm{Cr}$ & $\begin{array}{c}\text { USEPA:3050B, \& Chromium by Flame } \\
\text { AAS }\end{array}$ & $\mathrm{mg} / \mathrm{kg}$ \\
\hline 22 & Iron as $\mathrm{Fe}$ & $\begin{array}{l}\text { USEPA:3050B, \& Iron by UV-Vis } \\
\text { spectrophotometer }\end{array}$ & $\mathrm{mg} / \mathrm{kg}$ \\
\hline 23 & Lead as $\mathrm{Pb}$ & USEPA:3050B, \& Lead by Flame AAS & $\mathrm{mg} / \mathrm{kg}$ \\
\hline 24 & Organic Carbon & - & $\%$ \\
\hline
\end{tabular}




\section{Antimicrobial analysis}

\section{Bacteria}

- E. coli bacteria was obtained from the SNIST Biotechnology Department.

- E. coli was grown on the nutrient medium to obtain the mother culture.

- Antbacterial activity was conducted using various cow urine concentrations $(5 \%, 15 \%, 25 \%)$ Fungi using well diffusion method.

- A fungal species was isolated from a raw coconut. Based on its morphological character, it was tentatively identified as Aspergillus.

- Fungi was grown on the YPD medium to obtain the mother culture.

- Antifungal activity was conducted using various cow urine concentrations (5\%, 15\%, 25\%).

\section{Gas chromatography mass spectroscopy analysis}

Gas chromatography-mass spectroscopy analysis was conducted to determine the compounds present in the cow urine which may have made the changes in the plant growth, soil and antimicrobial properties.

1. The urine sample was extracted in dichloromethane using the principle of liquid-liquid extraction.

2. The cow urine was mixed with dichloromethane in 1:1 ratio and was left in shaker for $3 \mathrm{hrs}$.

3. The difference in their densities resulted in a two-layer liquid i.e top layer-cow urine, bottom layer-dichloromethane.

4. The dichloromethane was extracted using a pipette and was transferred into $2 \mathrm{ml}$ vials.

The protocol for GCMS [6]:

The GC-MS system started with the initial oven temperature of $60{ }^{\circ} \mathrm{C}$ for $5 \mathrm{~min}$, increasing to $240{ }^{\circ} \mathrm{C}$ at a rate of $15^{\circ} \mathrm{C}$ for $5 \mathrm{~min}$, and then to $300^{\circ} \mathrm{C}$ at a rate of $15^{\circ} \mathrm{C}$ for $5 \mathrm{~min}$. The injector temperature was maintained at $200^{\circ} \mathrm{C}$. The interface temperature was $250{ }^{\circ} \mathrm{C}$. Helium was used as a mobile phase at a flow rate of $1.0 \mathrm{~mL} / \mathrm{min}$. Mass spectral detection was carried out in electron ionization mode by scanning at $40-600(\mathrm{~m} / \mathrm{z})$.

The GCMS analysis was performed at IICT, Tarnaka.

\section{Results}

\section{Growth observation}

The plants were placed on white sheet of paper and the parameters were measured as per protocol mentioned in the methods. The growth observation of various plants is shown in Fig. 2, Fig. 3, Fig. 4, Fig. 5.

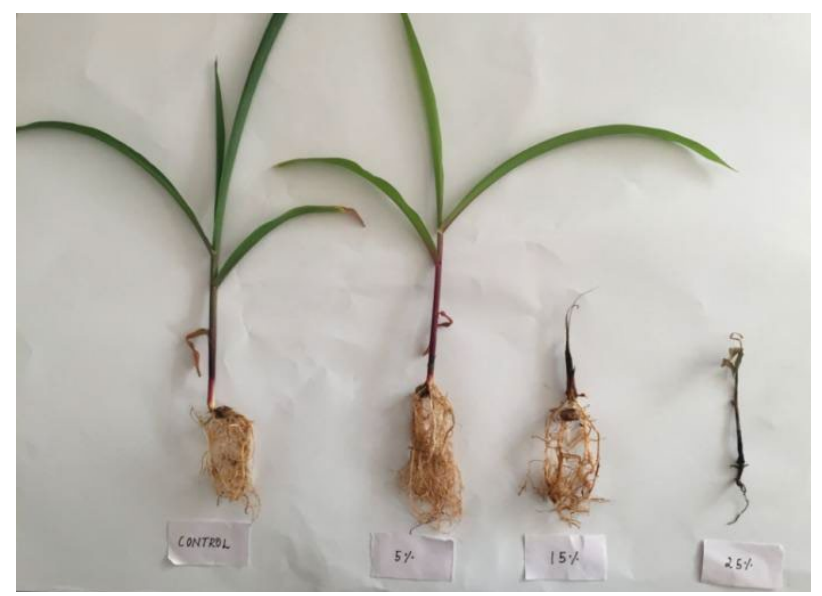

Figure 2. Growth of Zea Mays (MAIZE) in various concentrations 


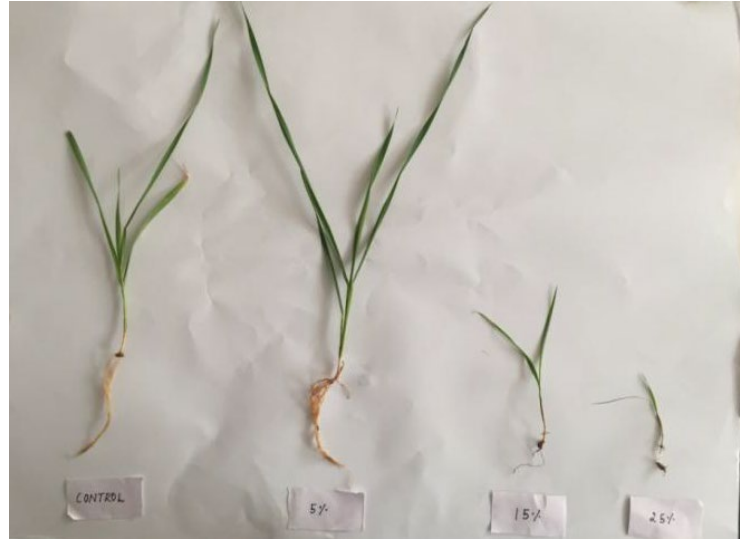

Figure 3. Growth of Cymbopogon citrates (GRASS) in various concentrations

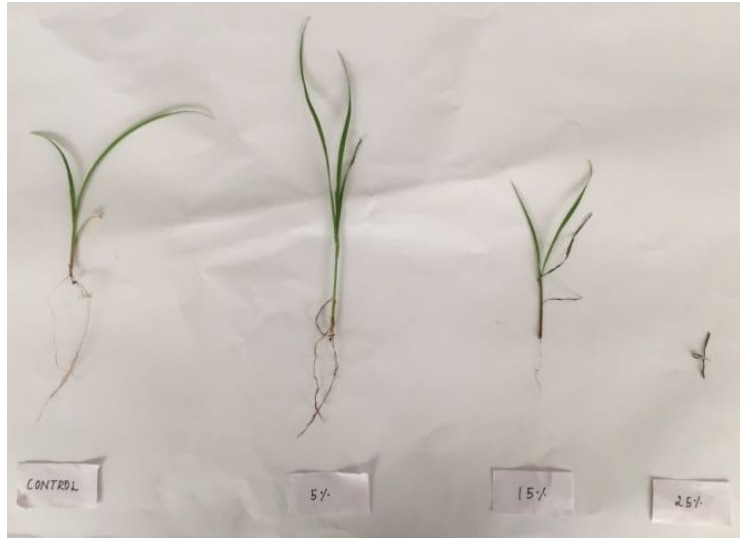

Figure 4. Growth of Triticum aestivum (WHEAT) in various concentrations

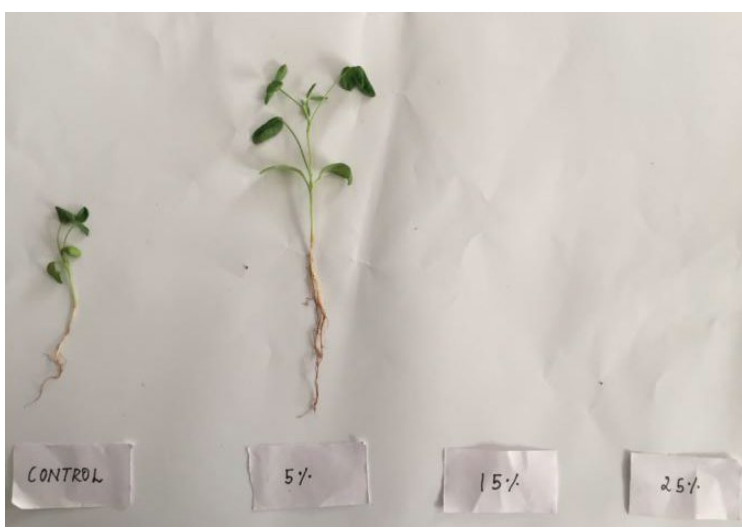

Figure 5. Growth of Trigonella foenum graecum (METHI) in various concentrations

There was no growth observed in (Ocimum basilicum)Tukmaria seeds.

The following seeds have not grown at particular cow urine concentrations

- (Ocimum basilicum) Tukmaria seeds- control

- (Ocimum basilicum)Tukmaria seeds-5\% concentration

- (Ocimum basilicum)Tukmaria seeds-15\% concentration

- ((Ocimum basilicum)Tukmaria seeds- $25 \%$ concentration

- (Trigonella foenum graecum) Methi seeds- $15 \%$ concentration

- (Trigonella foenum graecum) Methi seeds-25\% concentration

The following seeds have shown poor growth

- (Zea mays) Maize seeds- $15 \%$ concentration

- (Zea mays) Maize seeds-25\% concentration

- (Triticum aestivum) Wheat seeds-25\% concentration 
Physical parameters comparison

The plants have shown different physical changes with different cow urine concentrations. This shows that cow urine has effect on the growth of the plants.

The data from the physical parameters was tabulated in Table 2, Table 3, Table 4, Table 5.

Table 2. Data of physical parameters of Maize (Zea mays)

\begin{tabular}{|c|c|c|c|c|}
\hline Maize & Control & $\mathbf{5 \%}$ Concentration & $\mathbf{1 5 \%}$ Concentration & $\mathbf{1 5 \%}$ Concentration \\
\hline Chlorophyll A & 0.8711 & 0.2005 & -0.168 & 0.2304 \\
\hline Chlorophyll B & 0.5051 & 0.804 & -0.2105 & 0.4813 \\
\hline $\begin{array}{c}\text { Total } \\
\text { Chlorophyll }\end{array}$ & 0.4481 & 0.7145 & -0.02276 & 0.4471 \\
\hline Plant height-cm & 31.5 & 41.6 & 18.3 & 13.6 \\
\hline Root length-cm & 9 & 13.1 & 10.5 & 5 \\
\hline Shoot length-cm & 22.5 & 28.5 & 7.8 & 0.6 \\
\hline Leaf length-cm & 21.2 & 21.7 & 0 & 0 \\
\hline Leaf breadth-cm & 1.7 & 1.6 & 0 & 0 \\
\hline No. Of Branches & 4 & 4 & 0 & 0.11 \\
\hline Root weight-gm & 0.98 & 2.16 & 1.58 & 0 \\
\hline
\end{tabular}

Table 3. Data of physical parameters of Lemon grass (Cymbopogon citratus)

\begin{tabular}{|c|c|c|c|c|}
\hline Grass & Control & $\mathbf{5 \%}$ Concentration & $\mathbf{1 5 \%}$ Concentration & $\mathbf{1 5 \%}$ Concentration \\
\hline Chlorophyll A & 0.3787 & 0.7829 & 0.1587 & 0.1272 \\
\hline Chlorophyll B & 1.3882 & 2.3316 & 0.5284 & 0.1443 \\
\hline $\begin{array}{c}\text { Total } \\
\text { Chlorophyll }\end{array}$ & 1.2351 & 2.0794 & 0.4854 & 0.1687 \\
\hline Plant height-cm & 34.7 & 43.1 & 16.5 & 10.3 \\
\hline Root length-cm & 9.5 & 11.3 & 5.5 & 2.9 \\
\hline Shoot length-cm & 25.2 & 31.8 & 11 & 7.4 \\
\hline Leaf length-cm & 19.3 & 24.8 & 7.9 & 0.1 \\
\hline Leaf breadth-cm & 0.4 & 0.6 & 0.2 & 3 \\
\hline No. Of Branches & 4 & 4 & 3 & 0.03 \\
\hline Root weight-gm & 0.06 & 0.11 & 0.04 & 5 \\
\hline
\end{tabular}


Table 4. Data of physical parameters of Wheat (Triticum aestivum)

\begin{tabular}{|c|c|c|c|c|}
\hline Wheat & Control & $\mathbf{5 \%}$ Concentration & $\mathbf{1 5 \%}$ Concentration & $\mathbf{1 5 \%}$ Concentration \\
\hline Chlorophyll A & 1.5038 & 0.4236 & 0.6342 & -1.163 \\
\hline Chlorophyll B & 0.8055 & 0.9345 & 0.6788 & -1.9297 \\
\hline $\begin{array}{c}\text { Total } \\
\text { Chlorophyll }\end{array}$ & 0.8189 & 0.8356 & 0.6139 & -2.7919 \\
\hline Plant height-cm & 28.9 & 30.2 & 17.8 & 3 \\
\hline Root length-cm & 12.4 & 8.3 & 4.6 & 2.8 \\
\hline Shoot length-cm & 16.5 & 21.9 & 13.2 & 1.9 \\
\hline Leaf length-cm & 12.6 & 16.2 & 8.9 & 0.1 \\
\hline Leaf breadth-cm & 0.4 & 0.3 & 0.3 & 2 \\
\hline No. Of Branches & 4 & 5 & 4 & 0 \\
\hline Root weight-gm & 0.03 & 0.05 & 0.01 & \\
\hline
\end{tabular}

Table 5. Data of physical parameters of Methi (Trigonella foenum graecum)

\begin{tabular}{|c|c|c|c|c|}
\hline Methi & Control & $\mathbf{5 \%}$ Concentration & $\mathbf{1 5 \%}$ Concentration & $\mathbf{1 5 \%}$ Concentration \\
\hline Chlorophyll A & 0.3069 & 2.962 & 0 & 0 \\
\hline Chlorophyll B & 0.6915 & 5.929 & 0 & 0 \\
\hline $\begin{array}{c}\text { Total } \\
\text { Chlorophyll }\end{array}$ & 0.6159 & 5.2519 & 0 & 0 \\
\hline Plant height-cm & 10.8 & 17.9 & 0 & 0 \\
\hline Root length-cm & 5.6 & 9.2 & 0 & 0 \\
\hline Shoot length-cm & 5.2 & 8.7 & 0 & 0 \\
\hline Leaf length-cm & 1.4 & 2.2 & 0 & 0 \\
\hline Leaf breadth-cm & 0.6 & 0.9 & 0 & 0 \\
\hline No. Of Branches & 5 & 7 & 0 & 0 \\
\hline Root weight-gm & 0.03 & 0.01 & 0 & 0 \\
\hline
\end{tabular}

The graphs were plotted for each of the plant under various concentrations. The graphs are depicted in Fig. 6-Maize, Fig. 7-Grass, Fig. 8-Wheat, Fig. 9-Methi. 


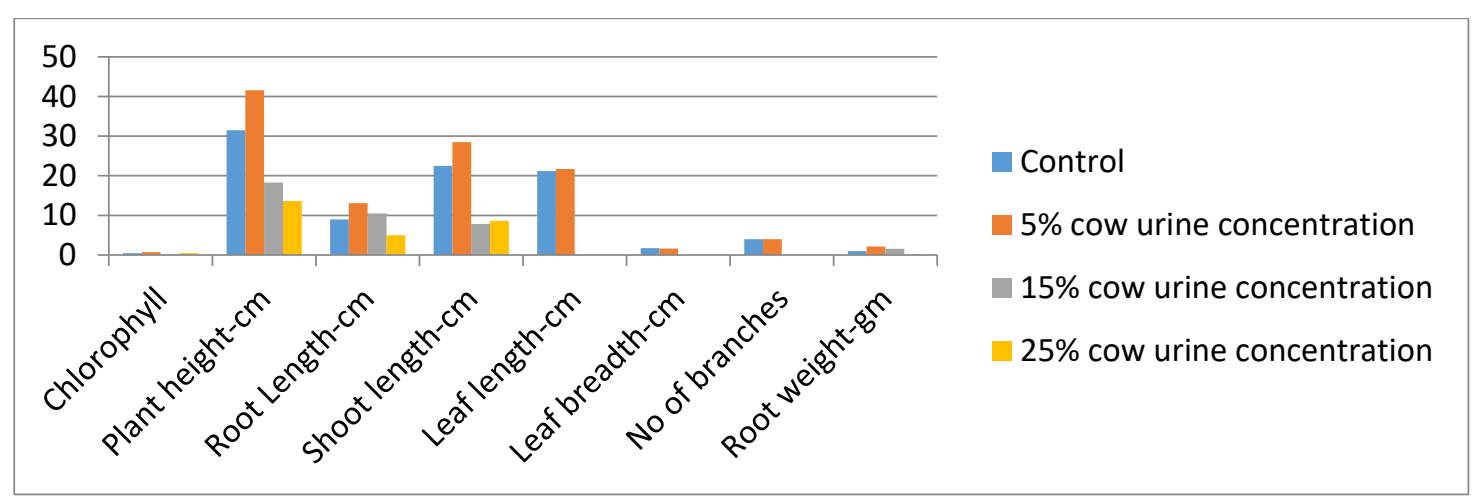

Figure 6. Graphical representation of physical parameters of (Zea mays) MAIZE on various concentrations

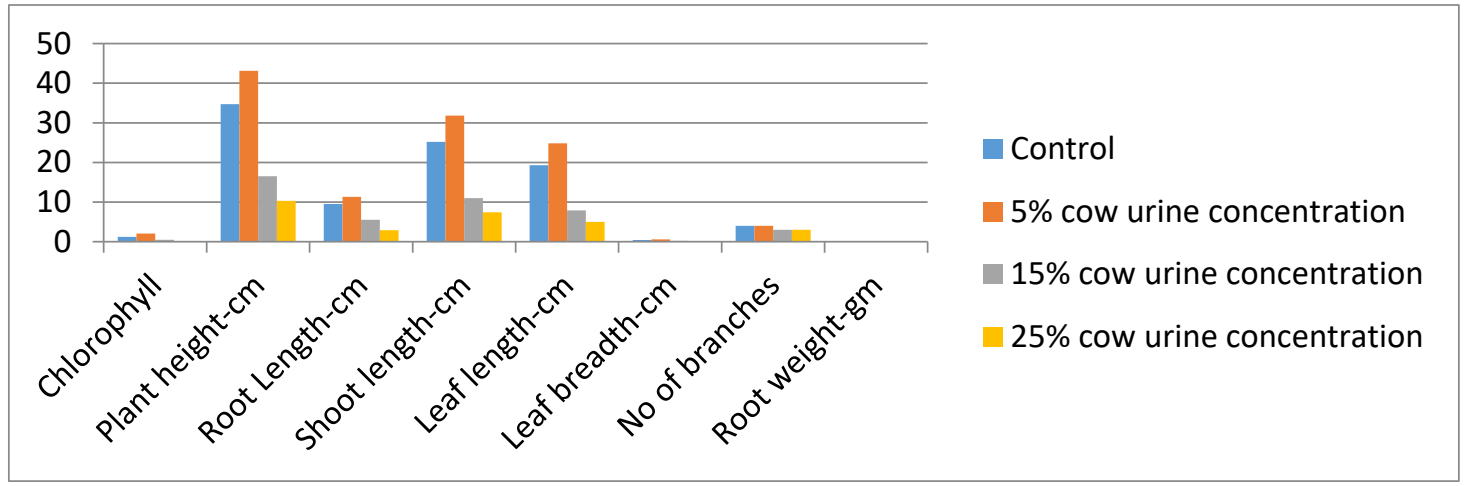

Figure 7. Graphical representation of physical parameters of (Cymbopogon citrates) GRASS on various concentrations

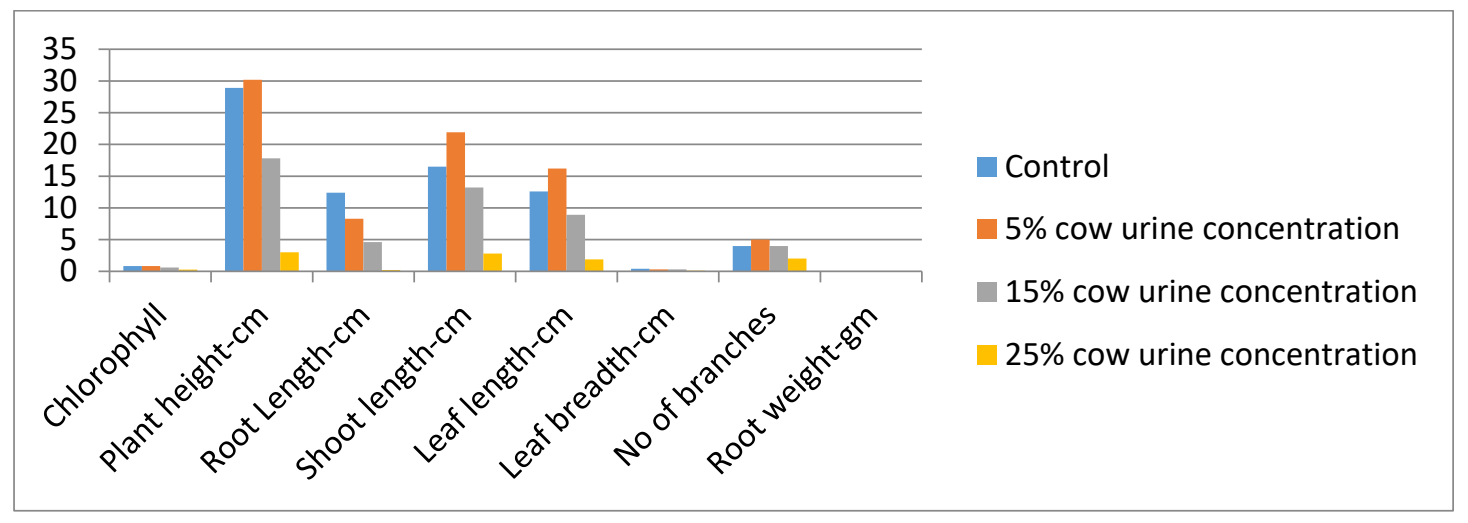

Figure 8. Graphical representation of physical parameters of (Triticum aestivum) WHEAT on various concentrations

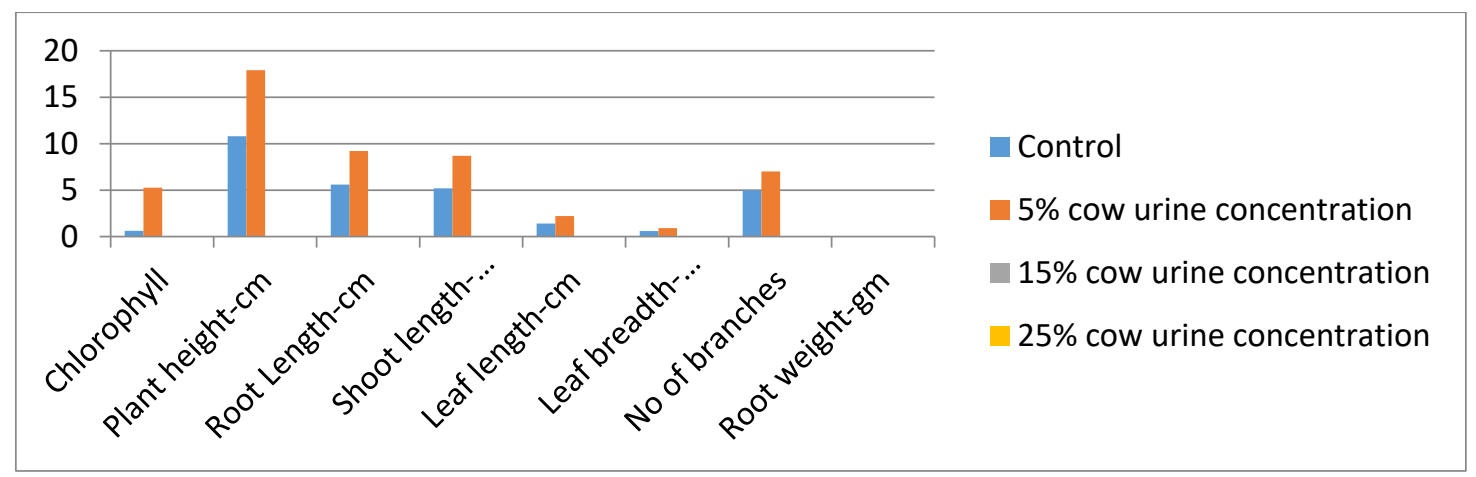

Figure 9. Graphical representation of physical parameters of (Trigonella foenum graecum) METHI on various concentrations 
The observations in the plant physical features depict that 5\% cow urine concentration has shown enhanced growth in the following plants:

- (Zea mays) Maize

- (Triticum aestivum) Wheat

- (Cymbopogon citrates) Grass

- (Trigonella foenum graecum) Methi

Data from soil analysis

The data from the soil analysis was tabulated in Table 6 .

Table 6. Data from soil analysis

\begin{tabular}{|c|c|c|c|c|}
\hline Parameters & Control & $5 \%$ cow urine & $15 \%$ cow urine & $25 \%$ cow urine \\
\hline $\mathrm{pH}$ & 8.75 & 8.58 & 8.38 & 7.85 \\
\hline $\begin{array}{c}\text { Electrical } \\
\text { Conductivity }\end{array}$ & 113.5 & 134.2 & 156.6 & 174.7 \\
\hline Bulk Density & 1.6 & 1.91 & 2.16 & 2.31 \\
\hline Moisture content & 3.41 & 7.82 & 12.81 & 15.6 \\
\hline Soil Texture-Sand & 10 & 7 & 8 & 13 \\
\hline Soil Texture-Silt & 28 & 12 & 12 & 14 \\
\hline Soil Texture-Clay & 62 & 81 & 80 & 73 \\
\hline Nitrogen & 91.05 & 95.42 & 93.28 & 91.08 \\
\hline $\begin{array}{l}\text { Phosphorus as } \\
\text { P2O5 }\end{array}$ & 20.31 & 18.27 & 17.99 & 18.76 \\
\hline Potassium as $\mathrm{K} 2 \mathrm{O}$ & 87.17 & 85.01 & 88.64 & 91.11 \\
\hline Sodium as $\mathrm{Na} 2 \mathrm{O}$ & 91.33 & 93.64 & 90.07 & 97.63 \\
\hline Calcium as $\mathrm{Ca}$ & 32.06 & 60.12 & 48.09 & 40.08 \\
\hline Magnesium as $\mathrm{Mg}$ & 16.98 & 4.83 & 4.92 & 16.98 \\
\hline Chlorides as $\mathrm{Cl}$ & 269.9 & 304.9 & 274.9 & 289.9 \\
\hline Sulphates as SO4 & 16.44 & 18.13 & 19.18 & 18.21 \\
\hline Copper as $\mathrm{Cu}$ & $<1.0$ & $<1.0$ & $<1.0$ & $<1.0$ \\
\hline Manganese & $<1.0$ & $<1.0$ & $<1.0$ & $<1.0$ \\
\hline Molybdenum & $<1.0$ & $<1.0$ & $<1.0$ & $<1.0$ \\
\hline Boran & $<1.0$ & $<1.0$ & $<1.0$ & $<1.0$ \\
\hline Zinc as $\mathrm{Zn}$ & 1.92 & 2.32 & 2.24 & 2.19 \\
\hline Chromium as $\mathrm{Cr}$ & $<1.0$ & $<1.0$ & $<1.0$ & $<1.0$ \\
\hline Iron as $\mathrm{Fe}$ & $<2.0$ & $<2.0$ & $<2.0$ & $<2.0$ \\
\hline Lead as $\mathrm{Pb}$ & $<2.0$ & $<2.0$ & $<2.0$ & $<2.0$ \\
\hline Organic Carbon & 0.34 & 0.37 & 0.35 & 0.39 \\
\hline
\end{tabular}


The following parameters have shown increase in content with increase in cow urine concentration:

- Moisture content

- Electrical conductivity

- Bulk density

- $\mathrm{pH}$

\section{Antibacterial tests}

The antibacterial tests were conducted using disc diffusion method and the results are depicted in Fig.10.
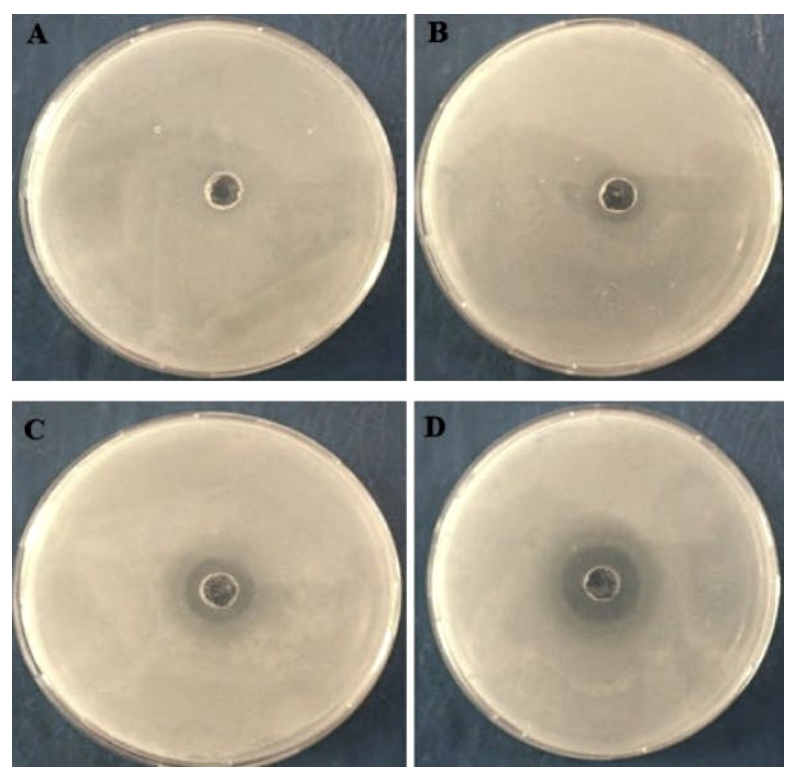

Figure 10. Antibacterial Activity of Cow urine on various concentrations: a) $0 \%$ concentration b) $5 \%$ concentration c) $15 \%$ concentration d) $25 \%$ concentration

The data from the antibacterial tests are shown in Table 7.

Table 7. Diameter of the inhibition zone in various concentrations

\begin{tabular}{|c|c|}
\hline Concentration & Diameter of the inhibition zone \\
\hline $0 \%$ cow urine concentration & $0.0 \mathrm{~cm}$ \\
\hline $5 \%$ cow urine concentration & $0.6 \mathrm{~cm}$ \\
\hline $15 \%$ cow urine concentration & $1.4 \mathrm{~cm}$ \\
\hline $25 \%$ cow urine concentration & $2.1 \mathrm{~cm}$ \\
\hline
\end{tabular}

From the data, it can be inferred that the diameter of the inhibition zone increases with increase in cow urine concentration. This depicts that cow urine acts as an antibacterial agent with increase in concentration.

\section{Antifungal tests}

The fungal sample was collected from a raw coconut (Fig. 11) and was inoculated in YPD medium which supports the growth of fungi. 


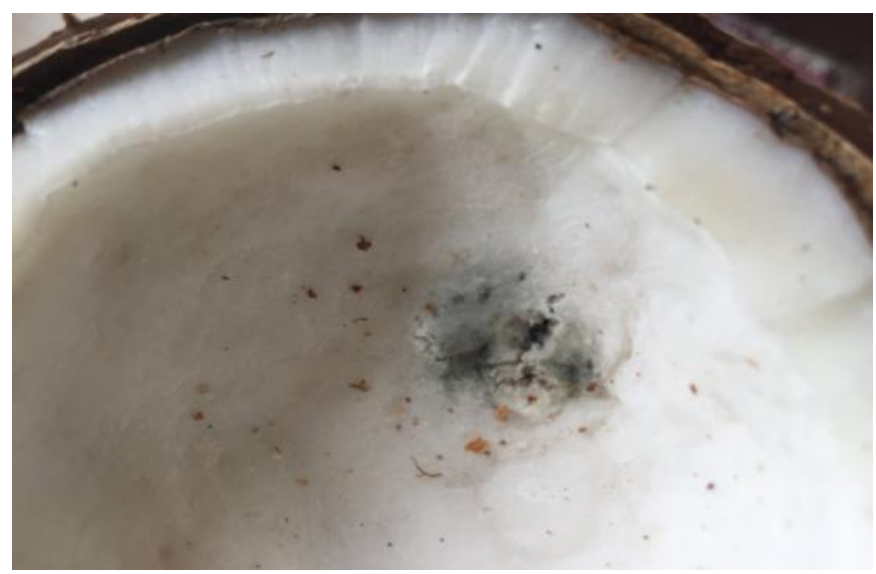

Figure 11. Growth of fungi on raw coconut

The mother culture was obtained by growing the fungi on YPD media-Fig. 12.

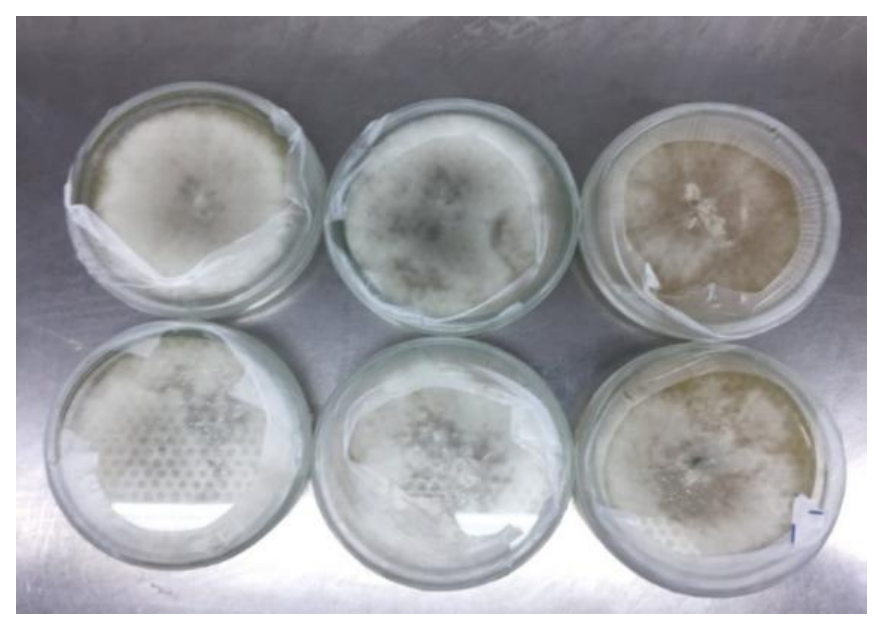

Figure 12. Fungi grown on YPD medium for mother culture

Using slide culture technique (Fig. 13), the fungus was identified as Aspergillus based on its morphological characteristics.

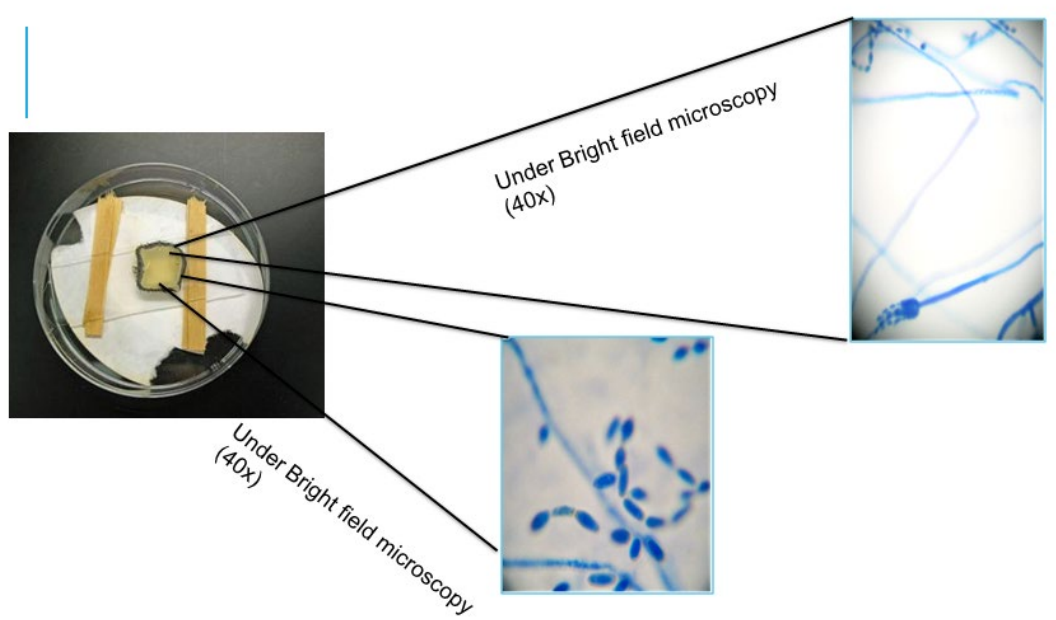

Figure 13. Slide culture technique for fungus

The antifungal tests were conducted by poisoning the media with cow urine. The cow urine concentrations i.e 5\%-Fig. 14, 15\%-Fig 15, 25\%-Fig 16, was added into the YPD media and was sterilized using autoclave. The fungi were inoculated on the media and was kept in an incubator for 6 days at a temperature of 30 Celsius. 


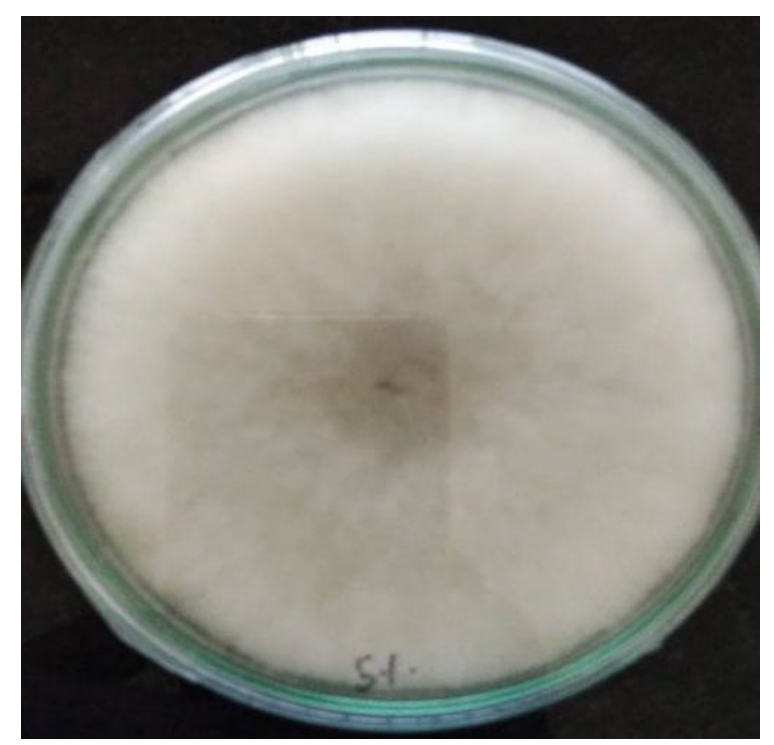

Figure 14. Antifungal test using 5\% concentration

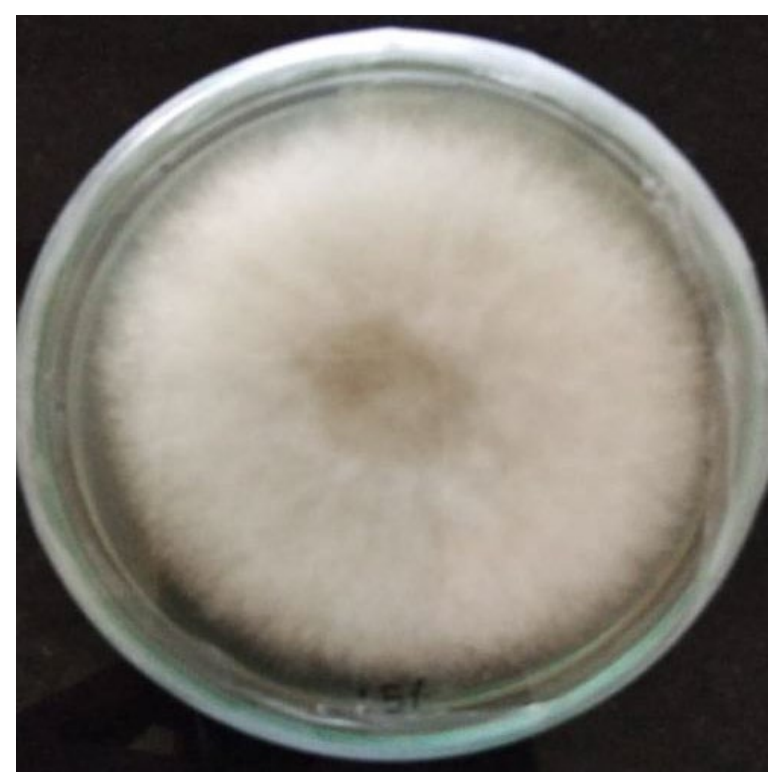

Figure 15. Antifungal test using $15 \%$ concentration

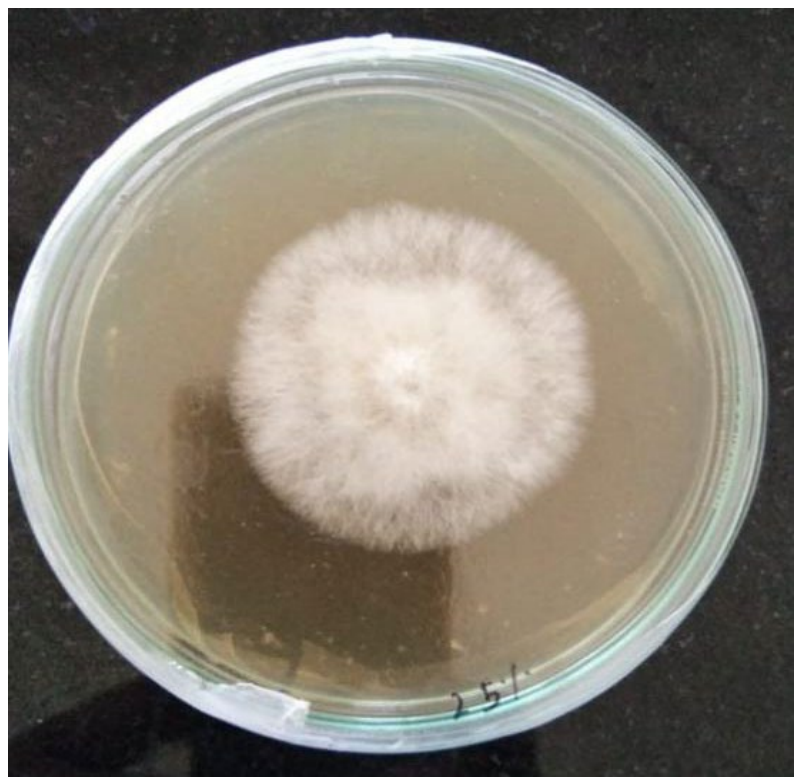

Figure 16. Antifungal test using 25\% concentration 
The results are shown in Table 8 .

Table 8. Diameter of fungi culture in various concentrations

\begin{tabular}{|c|c|}
\hline Concentration & Diameter of the fungi culture \\
\hline $0 \%$ cow urine concentration & $6 \mathrm{~cm}$ \\
\hline $5 \%$ cow urine concentration & $5.5 \mathrm{~cm}$ \\
\hline $15 \%$ cow urine concentration & $4.9 \mathrm{~cm}$ \\
\hline $25 \%$ cow urine concentration & $3.1 \mathrm{~cm}$ \\
\hline
\end{tabular}

The growth of fungi was inhibited with the increase in cow urine concentration.

Gas chromatography and mass spectroscopy analysis

The GCMS analysis was conducted as per the protocol mentioned in the methods and the graphs obtained.

The compounds identified in the analysis were,

1. 1H-Indole, 1-Methyl-2-Phenyl (Pubchem ID-77095)

2. Isophthaladehyde (Pubchem ID-34777)

3. Octane, 6-ethyl-2-methyl (Pubchem ID-537768)

4. 7-Methyl-Z-8,10-dodecadienal (Pubchem ID-5363533)

5. 1(3H)-Isobenzofuranone,3a,4,5,7a-tetrahydro-4-hydroxy-3a,7a-dimethyl-,

(3a.alpha.,4.beta.,7a.alpha.) - (.+/-.) (Pubchem ID- Not found)

6. Phenol, 2,4-bis(1,1-dimethylethyl) (Pubchem ID-528937)

7. Triacontane (Pubchem ID-12535)

8. L-leucine, N-(N-acetylglycyl)-butylester (Pubchem ID-Not found)

9. Tetradecanoic acid, trimethylsilylester (Pubchem ID-519592)

10. 1,2-Benzenedicarboxylic acid, butyl 2-ethylhexyl ester (Pubchem ID-6818)

11. Tridecane, 7-propyl (Pubchem ID-Not found)

12. N-Hexadecanoic acid (Pubchem ID-540086) fungicide

13. Dibutyl phthalate (Pubchem ID-3026) *Drug*

14. Hexadeconic acid, trimethylsilyl ester (Pubchem ID- 521638)

15. 4-(p-methoxyphenyl)-3-buten-2-one, thiosemicarbazone (Pubchem ID-9603450)

16. 1-cyclohexyldimethylsilyloxy-3,5-dimethyl benzene (Pubchem ID-532617) Table 9.

The following compounds (16) were identified and their properties are mentioned in the 
Table 9. Pubchem ID, Properties and References of the identified compounds

\begin{tabular}{|c|c|c|c|}
\hline Pubchem ID & Name of compound & Properties & Reference \\
\hline 77095 & $\begin{array}{l}\text { 1H-Indole, 1-Methyl-2- } \\
\text { Phenyl }\end{array}$ & Antifungal & https://doi.org/10.14233/ajchem.2017.20423 [7] \\
\hline 34777 & Isophthaladehyde & $\begin{array}{l}\text { Antifungal, } \\
\text { Antibacterial }\end{array}$ & https://www.ncbi.nlm.nih.gov/pubmed/1953927[8] \\
\hline 537768 & $\begin{array}{l}\text { Octane, 6-ethyl-2- } \\
\text { methyl }\end{array}$ & - & No information available about this compound \\
\hline 5363533 & $\begin{array}{l}\text { 7-Methyl-Z-8,10- } \\
\text { dodecadienal }\end{array}$ & - & No information available about this compound \\
\hline Not found & $\begin{array}{c}1(3 \mathrm{H})- \\
\text { Isobenzofuranone,3a,4, } \\
\text { 5,7a-tetrahydro-4- } \\
\text { hydroxy-3a,7a- } \\
\text { dimethyl-,(3a.alpha.,4.b } \\
\text { eta.,7a.alpha.)-(.+/-.) }\end{array}$ & - & No information available about this compound \\
\hline 528937 & $\begin{array}{l}\text { Phenol, 2,4-bis(1,1- } \\
\text { dimethylethyl) }\end{array}$ & Antifungal & https://doi.org/10.1016/j.jplph.2013.07.004 [9] \\
\hline 12535 & Triacontane & $\begin{array}{l}\text { Plant growth } \\
\text { regulator }\end{array}$ & https://doi.org/10.1080/17429145.2011.619281 [10] \\
\hline Not found & $\begin{array}{l}\text { L-leucine, } \mathrm{N}-(\mathrm{N}- \\
\text { acetylglycyl)-butylester }\end{array}$ & - & No information available about this compound \\
\hline 519592 & $\begin{array}{l}\text { Tetradecanoic acid, } \\
\text { trimethylsilylester }\end{array}$ & $\begin{array}{l}\text { Antifungal, } \\
\text { Antibacterial }\end{array}$ & https://doi.org/10.1002/ardp.19963291102 [11] \\
\hline 6818 & $\begin{array}{l}1,2- \\
\text { Benzenedicarboxylic } \\
\text { acid, butyl 2-ethylhexyl } \\
\text { ester }\end{array}$ & Antifungal & $\begin{array}{c}\text { http://shodhganga.inflibnet.ac.in/bitstream/10603/1 } \\
\text { 84541/19/6_abstract.pdf }\end{array}$ \\
\hline 246301 & Tridecane, 7-propyl & - & No information available about this compound \\
\hline 540086 & N-Hexadecanoic acid & $\begin{array}{l}\text { Antifungal, } \\
\text { Antibacterial }\end{array}$ & $\begin{array}{c}\text { https://doi.org/10.7164/antibiotics.39.888 [12] } \\
\text { https://doi.org/10.1111/j.1747-0285.2012.01418.x } \\
{[13]}\end{array}$ \\
\hline 3206 & Dibutyl phthalate & Medicinal oil & https://doi.org/10.1186/s40199-014-0078-1 [14] \\
\hline 521638 & $\begin{array}{l}\text { Hexadeconic acid, } \\
\text { trimethylsilyl ester }\end{array}$ & - & No information available about this component \\
\hline 9603450 & $\begin{array}{l}\text { 4-(p-methoxyphenyl)- } \\
\text { 3-buten-2-one, }\end{array}$ & $\begin{array}{l}\text { Plant growth } \\
\text { regulator }\end{array}$ & $\begin{array}{c}\text { http://agris.fao.org/agris- } \\
\text { search/search.do?recordID=XB8320803 [15] }\end{array}$ \\
\hline 532617 & $\begin{array}{l}1- \\
\text { cyclohexyldimethylsilyl } \\
\text { oxy-3,5-dimethyl } \\
\text { benzene }\end{array}$ & - & No information available about this compound \\
\hline
\end{tabular}

Out of the following compounds, 6 compounds were identified as antifungal compounds, 3 were identified as antibacterial compounds and 2 compounds were identified as plant growth regulators. 


\section{Discussion}

The plants were grown using different concentrations (control $(0 \%), 5 \%, 15 \%, 25 \%)$ of cow urine for 30 days and the growth was observed. The plants showed varied growth at different concentrations and the physical parameters were measured and tabulated. According to the measurements, the 5\% cow urine concentration showed more growth when compared to other concentrations. The soil was sent for soil analysis to check for the changes made to the soil by cow urine. The various parameters of the soil were analysed. The parameters i.e $\mathrm{pH}$, Bulk density, Electrical conductivity, Moisture content were increased with increase in cow urine concentrations. The antimicrobial properties of the cow urine were examined. The antibacterial test was conducted on E. coli using disc diffusion method. The inhibition zone increased with increase in cow urine concentration indicating the presence of antibacterial compounds in cow urine. The fungus was isolated from raw coconut and slide culture technique was used to determine its morphological features. Based on its morphological features, it was identified as Aspergillus. The antifungal tests were conducted by poisoning the media with various cow urine concentrations and inoculating the fungi. The diameter of the fungi culture decreased with increase in cow urine concentration indicating the presence of antifungal agents. Gas chromatography mass spectroscopy analysis was performed to identify the compounds in the cow urine. The cow urine was extracted by liquid-liquid phase extraction using dichloromethane and was sent for analysis. 16 compounds were identified in the sample and their respective pubchem IDs were searched. 2 compounds didn't have any pubchem ID indicating the presence of new compounds. The compounds were searched for their properties using literature review. Out of 16 compounds, 6 compounds were identified as antifungal, 3 compounds were identified as antibacterial, and 2 compounds as plant growth enhancers.

From the above study, we can conclude that cow urine has the properties of plant growth enhancer and antimicrobial properties. Most of the compounds identified were produced synthetically. This can be avoided by extracting the selected compounds from the cow urine which can save money as well as protect our environment by avoiding the synthetic means of production.

\section{Acknowledgement}

This research was conducted in Sreenidhi Institute of Science and Technology, as a part major project. I would like to thank, Dr Bishwambhar Mishra for guiding us. I thank IICT, Tarnaka for allowing us to use their resources i.e Gas chromatography and mass spectroscopy and ECOICONS, ECIL for soil analysis.

\section{Conflict of interest}

The authors declare that they don't have any conflict of interest.

\section{References}

[1] Anuradha Verma, Babita Kumar, Manish K Singh, M D Kharya., Immunomodulatory potential of Cow urine, Der pharmacia Lettre, 3(2): (2011) 507-513.

[2] Ipsita mohanty, Manas Ranjan Senapati, Deepika Jena, Santwana Palai., Diversified used of cow urine, International Journal of Pharmacy and Pharmaceutical Sciences, 6(3): (2014) 22-29.

[3] Awale, Suresh \& Linn, Thein \& Myint Than, Myint \& Maung Thet, Maung \& Swe, Thein \& Saiki, Ikuo \& Kadota, Shigetoshi. An amazing cow's urine therapy practice in Myanmar. Journal of Traditional Medicines, 23: (2006) 178-183.

[4] Dhama, Kuldeep, Khurana, Sandip, Karthik K Tiwari, Ruchi, Malik, Yashpal RS Chauhan. Panchgavya: Immune-enhancing and Therapeutic Perspectives. Journal of Immunology and Immunopathology, 16: (2014) 1-11. 
[5] Neyak Sumanta, Choudhury Imranul Haque, Jaishee Nishika, Roy Suprakash., Spectrophotometric Analysis of Chlorophylls and Carotenoids From Commonly Grown Fern Species By Using Various Extracting Solvents, 4(9): (2014) 63-69.

[6] Syed Rizwan Ahamad, Abdul Qader Alhaider, Mohammad Raish, Faiyaz Shakeel., (2017), Matabolic and elemental analysis of camel and bovine urine by GC-MS and ICP-MS, 24(1) (2017) 23-29.

[7] Geetika Arora, Sunita Sharma, Sukesha Joshi., Synthesis of Substituted 2-Phenyl-1H-indoles and their Fungicidal Activity, Asian Journal of Chemistry, 2017, Vol 29, No. 8

[8] V Diurno, M Mazzoni, O \& Piscopo, E \& Bolognese, Adele. 2010. ChemInform Abstract: Synthesis and Antimicrobial Activity of 1,3,4-Triaryl-2-azetidinones. Farmaco (Società chimica italiana: 1989), Vol 4, Page no. 239-47

[9] GerardoRangel-Sánchez, EldaCastro-MercadoErnesto, García-Pineda., Avocado roots treated with salicylic acid produce phenol-2,4-bis (1,1-dimethylethyl), a compound with antifungal activity. Journal of Plant Physiology, (2013), Vol 171, Issues 3-4, Pages 189-198

[10] M Naeem, M Masroor A Khan, Moinuddin, Triacontonol: a potent plant growth regulator in agriculture, Journal of Plant Interactions, (2011), Volume 7 Issue 2, Page no 123-129

[11] Keykavous Parang, Edward E. Knaus, Leonard I. Wiebe, Soroush Sadari, Mohsen Daneshtalab Ferenc Csizmadia., Synthesis and antifungal activities of Myristic acid analogs, Arch Pharm, (1996) Vol 329, Issue 11, Page no 01-08

[12] Nongnuch Vanittanakom, Wolfgang Loeffler, Ulrike Koch, Günther jung, Fengycin-A Novel Antifungal lipopeptide antibiotic produced by bacillus subtilis F-29-3, The journal of antibiotics, (1986) Vol 39, Page no 99-104

[13] Vasudevan Aparna, Kalarickal V. Dileep, Pradeep K. Mandal, Ponnuraj Karthe, Chittalakkottu Sadasivan, Madathilkovilakathu Haridas. Anti-Inflammatory Property of n-Hexadecanoic Acid: Structural Evidence and Kinetic Assessment, Chemical Biology \& Drug Design, (2012) Vol 80, Issue 3, Page no 434-439

[14] Azadeh Manayi, Mahdieh Kurepaz-mahmoodabadi, Ahmad R Gohari, Yousef Ajani and Soodabeh Saeidnia, Presence of phthalate derivatives in the essential oils of a medicinal plant Achillea tenuifolia, DARU Journal of Pharmaceutical Sciences, (2014) Vol 22, Page no 78-85

[15] Pornchai Chuthamas. Effects of alpha cyclo-propyl-alpha-(4-methoxyphenyl-)-5pyrimidinemethanol [ancymidol] on the marigold cv. Sovereign [1981] Agricultural Information Bank of Asia, South-East Asian Regional Center for Graduate Study and Research in Agriculture. 